\title{
Early Mobilisation of Patients with Cervical Cord Injury Using the Halo Brace Device
}

H. Parry, BSc, MB, ChB, M. Delargy, MRCPI A. Burt, FRCP, Ed

Yorkshire Regional Spinal Injuries Centre, Pinderfields General Hospital, Aberford Road, Wakefield, West Yorkshire, UK.

\section{Summary}

The halo brace device for cervical spine stabilisation has been in use predominantly in North America since 1959. It has not yet found widespread use for the management of the spinal cord injured in the UK. At the Yorkshire Regional Spinal Injuries Unit we have used the device over 2 years on 20 patients with complete and incomplete traumatic cervical spinal cord injuries, and compared our results with those for the previous 20 patients with similar injuries treated with skull calipers and bed rest.

Patients using a halo brace device begin more active rehabilitation earlier as they are mobilised on average 5 weeks earlier than those whose traction is administered via skull calipers.

Halo brace patients begin weekend leave usually within 7 weeks of their injury compared to 14 weeks post-injury for caliper treated patients and were discharged on average 2 months earlier than the comparison group.

We believe that earlier mobilisation, weekend leave and discharge for halo brace patients in the absence of any neurological deterioration afford significant physical, psychological and management benefits.

Key words: Halo-brace; Quadriplegia; Rehabilitation; Skull calipers.

A halo brace device for stabilisation of the cervical spine was first used in 1959 (Perry, Nickel, 1959) to keep the cervical spine immobile after operative fusion. In 1960 it was first used as an immobilisation device following trauma to the cervical spine (James, 1960). Further studies in the USA and Canada, (Chan et al., 1983 and Tator et al., 1982) have shown that with care there are relatively few problems although it has been suggested that the halo brace is contra-indicated in patients with complete lesions (Cooper et al., 1979). Commonly, British practice following cervical fracture dislocation has been to apply skull calipers at the time of admission and reduce any dislocation with traction. This is followed by up to 3 months immobilisation with calipers and 
bed rest. This paper reports the use of the halo brace device in patients with cervical fractures and neurological injury, and a comparison with our previous patients using calipers.

\section{Method}

As soon as possible after transfer to the Spinal Injuries Unit at Pinderfields Hospital, Wakefield, patients with a cervical bony injury have a halo device fitted. If skull calipers have already been fitted elsewhere they are removed. The hair is shaved off around the area of the halo and the skin cleaned. The halo is positioned around the head using flat ended pins. Local anaesthetic is injected into the scalp around the areas where the four fixing pins are to be sited. The pins are then screwed in without the need for a formal incision and, keeping the halo symmetrical, are tightened to a torque of 6 inch pounds. The torque is checked 1 hour after application and again the next day. Thereafter the pins are tightened only if they become obviously loose or if the patient complains of pain around the pin site which is often the first symptom of a loose pin. The pin sites are cleaned daily and Cicatrin Powder dusted around their bases.

With the use of a $\mathrm{V}$ shaped bar between two sides of the halo, traction can be applied in the standard manner to reduce dislocation and we have used weights of up to $55 \mathrm{lbs}$. If reduction fails or the spine is markedly unstable then operative reduction and fixation is performed.

Soon after the dislocation is reduced the sheepskin lined thoracic brace is connected to the halo. X-Rays of the cervical spine are taken after application of the jacket while the patient is supine and later in the sitting position. We try to ensure that the front poles of the scaffolding are behind the visual field as bars within this area tend to make the patient feel incarcerated. Once the check XRay in the sitting position is deemed satisfactory a full active rehabilitation programme commences. The skin underneath the jacket, if anaesthetic, is checked daily, special care being taken over the scapulae which is a difficult area to visualise.

Relatives are involved from an early stage and shown how to handle the patient wearing a jacket and halo. They are also taught to give sub-cutaneous Heparin so that this can be continued at home. Once the relatives are competent and the home is suitable the patient is allowed on day or weekend leave. Providing no complications arise we prefer to keep the halo and jacket on for 10 to 12 weeks after which no further cervical support is necessary.

\section{Results}

In the 2 years we have been using the halo brace device for patients with cervical bony and neurological injury 23 have been referred in the early days following injury. Three of these were unsuitable for the use of the halo brace device. One had an associated fractured clavicle, one had abdominal trauma and the third patient had a rising neurological level. The remaining 20 patients had the halo fitted and this was used to reduce the dislocation in all but 2 cases. Eight patients had complete cord injuries and 12 incomplete. (Fig. 1). The 


$\begin{array}{ccc}\text { Grade } & \text { Halo } & \text { Caliper } \\ \text { A } & 8 & 8 \\ \text { B } & 2 & 3 \\ \text { C } & 3 & 3 \\ \text { D } & 3 & 3 \\ \text { E } & 4 & 3\end{array}$

Figure 1 Degree of completeness of the neurological lesion.

\begin{tabular}{|c|c|c|c|c|}
\hline \multirow[b]{2}{*}{ Level } & \multicolumn{2}{|c|}{ Halo } & \multicolumn{2}{|l|}{ Caliper } \\
\hline & Incomplete & Complete & Incomplete & Complete \\
\hline Odontoid/C1 & 1 & & 1 & \\
\hline C2 & 1 & & 1 & \\
\hline C3 & 1 & & 2 & \\
\hline C4 & 1 & 3 & & 3 \\
\hline C5 & 4 & & 4 & 2 \\
\hline C6 & 3 & 3 & 3 & 3 \\
\hline C7 & 1 & 2 & 1 & \\
\hline
\end{tabular}

Figure 2 Level of injury.

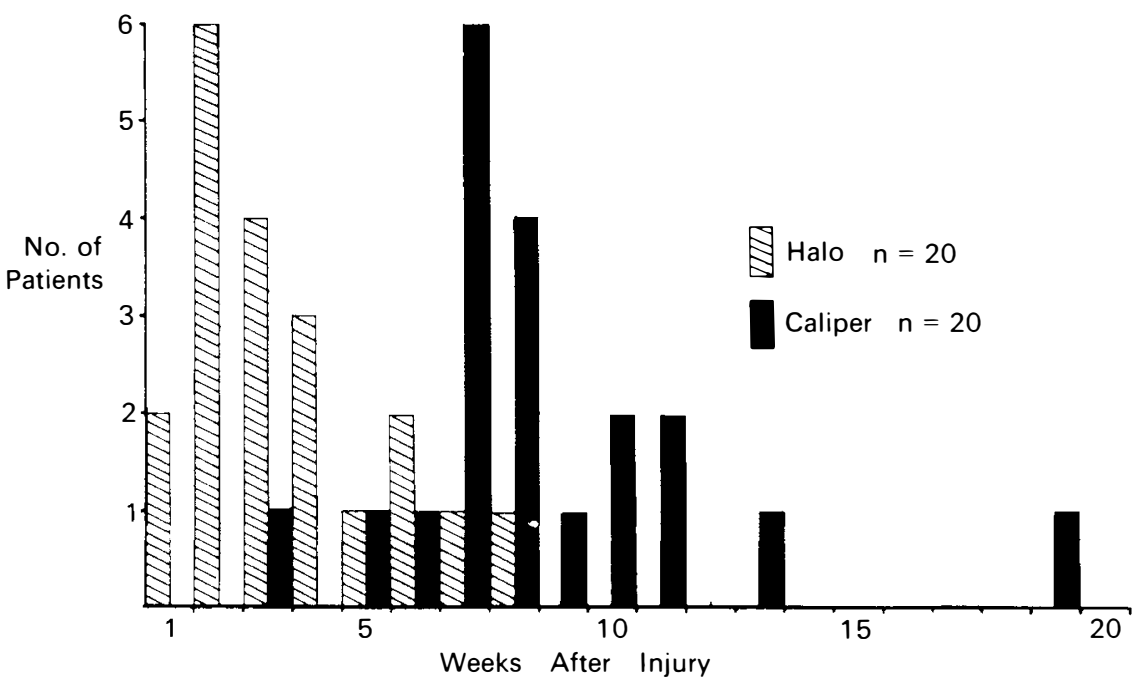

Figure 3 Mobilisation of patients first day up.

vertebral injuries ranged from odontoid to C7. (Fig. 2). The patients age ranged from 15 to 45 .

Two patients who failed to reduce with traction alone, one up to $50 \mathrm{lbs}$, underwent operative reduction and fixation. Three patients underwent elective fixation prior to being fitted with a jacket. For 3 patients in whom there was a later deterioration in fracture alignment, though without any neurological deterioration, operative fixation was performed. There is no need to remove the halo ring during operation and in fact the Surgeon found it a useful handle when manipulating the neck.

One patient had ventilatory problems which delayed application of the jacket until the 53rd day, otherwise there has been little problem in mobilising patients in jackets at an early stage (Fig. 3). We now have 11 patients who have been mobilised within 2 weeks of their accident or operation and in one case as early as 3 days. The jacket was removed from one patient after 15 days because of a breakdown of a surgical wound over the posterior iliac crest. The use of the jacket was unrelated. Two patients developed superficial skin grazes over the scapulae and to be absolutely safe the jackets were removed. Both these patients 


\begin{tabular}{lrccccc} 
& \multicolumn{2}{c}{ Complete } & \multicolumn{2}{c}{ Incomplete } & \multicolumn{2}{c}{ All patients } \\
& Halo & Caliper & Halo & Caliper & Halo & Caliper \\
Mobilised & 20 & 60 & 22 & 55 & 22 & 58 \\
Home & 49 & 100 & 42 & 76 & 45 & 86 \\
Discharge & 252 & 314 & 90 & 146 & 150 & 213
\end{tabular}

Figure 4 Rehabilitation and discharge home of patients.

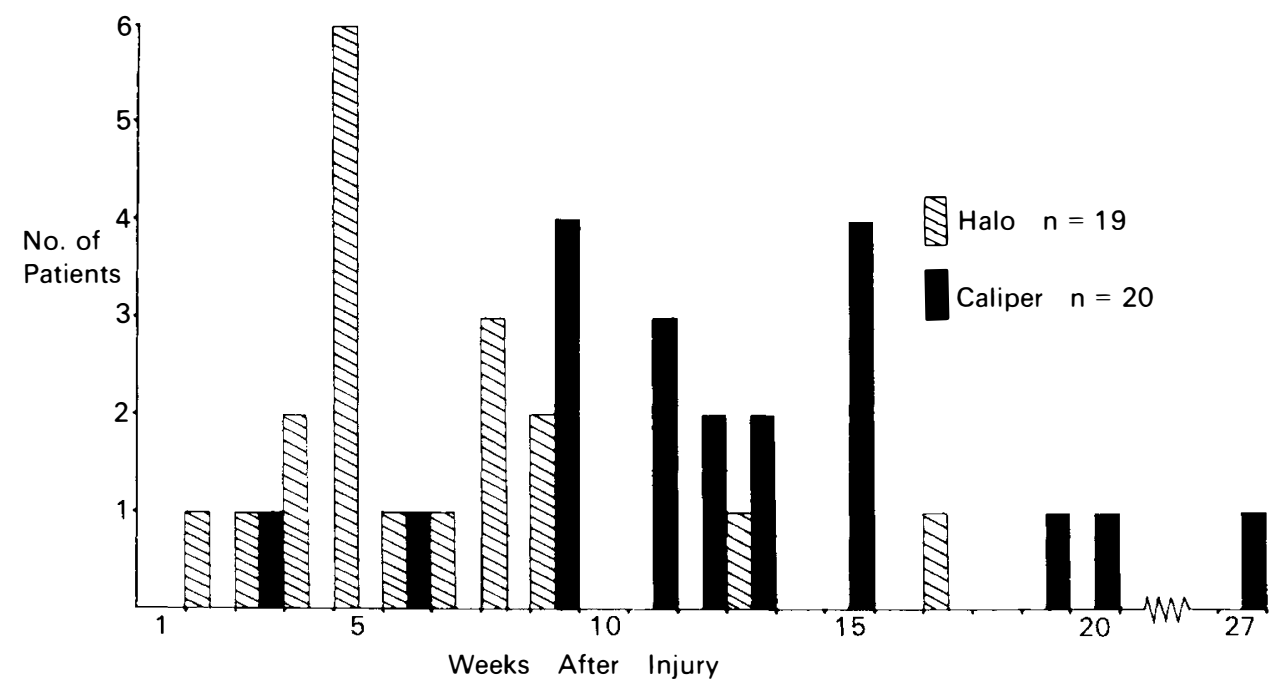

Figure 5 Time interval before first weekend home.

were over 8 weeks post-injury so continued mobilisation was possible using a SOMI collar. The halo was re-sited in 2 patients in whom pin slippage occurred, 1 on traction and the other due to incorrect lifting by the jacket. Two patients developed pin site infections which were treated by inserting another pin through an adjacent hole before removing the pin from the infected site. There is no need to remove the halo or jacket during this procedure. Necrosis around the pin site occurred in 1 patient and was dealt with in the same way as infection. Pain associated with pin sites, despite altering their position, led to removal of the halo ring and jacket from 3 patients at 5, 7 and 8 weeks respectively. Again these patients continued to be mobilised in SOMI collars. The remaining 14 patients had the halo brace for more than 10 weeks when the jacket was removed, and they required no further neck support. Postural hypotension sufficient to cause loss of consciousness during the initial mobilisation occurred in 2 patients who were both tall and thin.

Rehabilitation in the gymnasium began on an average at 3 weeks post injury in patients with complete and incomplete injuries. (Fig. 4). Difficulties with early mobilisation in the jacket were minor. Sitting balance took slightly longer to achieve as no neck movements were possible and dressing practice had to be postponed. Patients wearing the halo brace device went home for leave on an average within 7 weeks of their injury. Some patients with incomplete lesions 


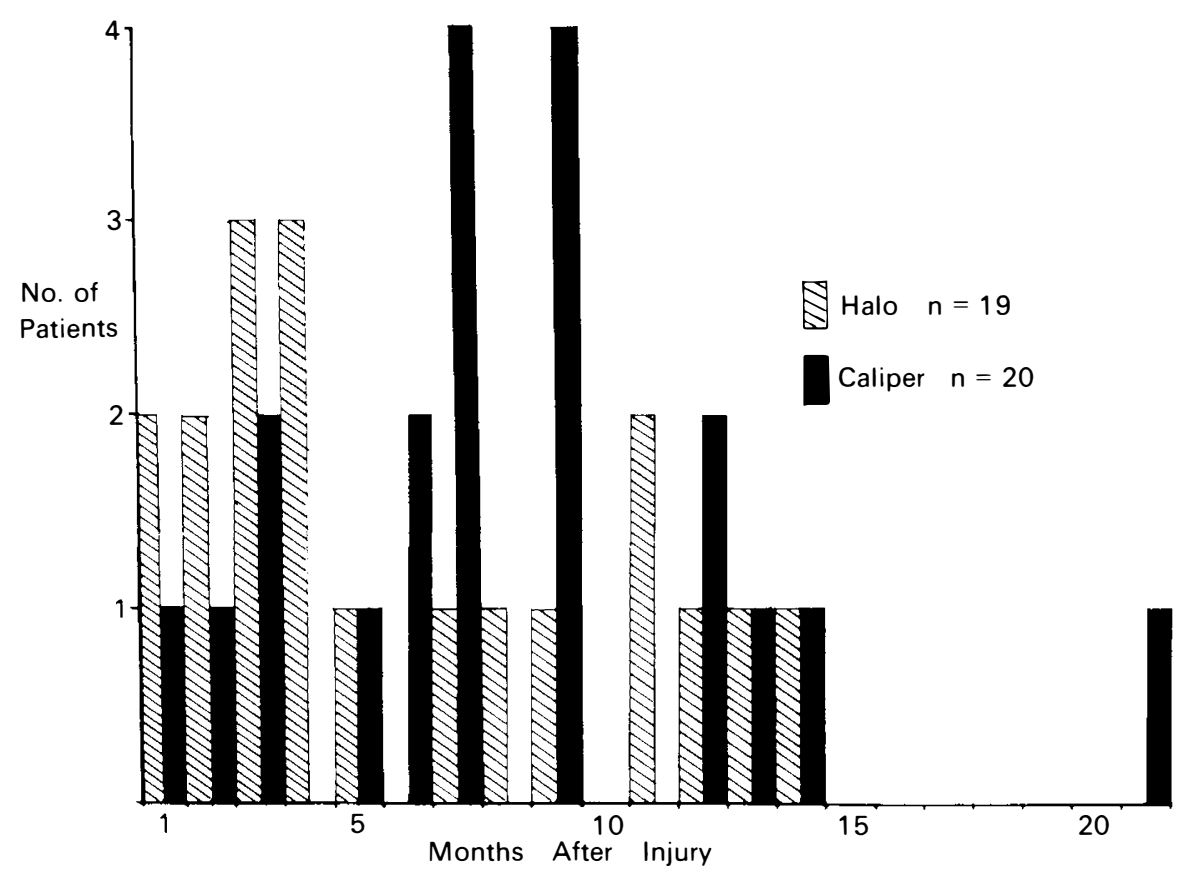

Figure 6 Final discharge home.

were discharged home at 2 weeks and 1 patient with a complete C6 quadriplegia was able to go home for weekend leave at 33 days post injury (Fig. 5). The only delay in discharging patients finally was the unsuitability of their accommodation. No patient or relative has yet complained of difficulty in coping with the halo brace device at home. In fact, most patients feel safer with this device fitted than when they were on bed rest with regular turning.

Patients with incomplete lesions who made a good neurological recovery and could walk were discharged home as early as 18 days wearing their halo brace device. Out-patient physiotherapy was continued at their local hospitals but they were reviewed at the spinal unit each week to check the position of the halo brace device. We have not encountered any halo brace related difficulties with any of these patients. Seven patients with complete neurological injury have been discharged between 109 to 376 post-injury. The other complete patient was transferred in the halo brace to his local spinal unit (Fig. 6).

A comparative retrospective survey of patients with similar injuries treated in this Unit with skull calipers and bed rest was carried out. In this group of 20 patients, 2 had the calipers slip requiring re-application and 4 developed pin site infections requiring complete removal and re-application. Mobilisation and active rehabilitation was started on average at 8 weeks with incomplete lesions, and $8 \frac{1}{2}$ weeks with complete lesions. Patients treated with calipers, on average, did not have their first weekend leave until 8 weeks for incomplete lesions and 14 weeks for complete lesions. Discharge for patients with complete lesions was, on average, 314 days (range 195-583). 


\section{Discussion}

The use of the halo brace device following spinal cord injury in this Unit has led to much earlier mobilisation, usually at approximately 3 weeks, compared with an average of over 8 weeks in those treated with calipers. In patients with uncomplicated injuries those with complete lesions can be mobilised as early as 2 weeks even when they have required stabilisation by spinous process wiring. With early mobilisation patients start more active physiotherapy and rehabilitation earlier and we believe this leads to less wasting of non-paralysed muscles and faster rehabilitation. Patients wearing the halo brace device report that they feel safer than when they are on traction and bed rest. They are able to sit in a wheelchair and relatives and friends can participate in their care at an earlier stage. The patient therefore experiences less isolation and is less dependent on professional nursing care. This is consolidated by weekends at home; usually within 7 weeks of injury an often earlier in incomplete quadriplegia. The halo and jacket have not proved to be a hindrance to patients going home. Morale appears to be maintained and the planning necessary to achieve an independent future can often be appreciated sooner by the patient and relatives compared with caliper treated patients who spend their first 2 months post-injury confined to their beds.

An early visit to the home by the patient with the Nursing Liaison Officer and Occupational Therapist leads to an earlier request for adaptations. This may be one of the reasons why patients with a complete cervical cord injury treated with halo brace device, on average, have been discharged 2 months earlier than the group treated with calipers. Discharge in these patients is usually related to the time when the adaptations have been made at home rather than when the patient has completed rehabilitation.

Patients who have good neurological recovery and are able to walk can be discharged safely with a halo brace fitted and followed up at Out-Patients. There has been no increase in neurological deficit as a result of using the halo brace device and late instability has not been detected. Postural hypotension is not found more often in patients with the halo brace device than in those treated by calipers. Infections around the pin sites and loose pins were no more prevalent with the halo, even for patients at home, and any halo pin problems were much more easily dealt with.

In 3 patients partial loss of alignment (flexion) in the cervical spine occurred following mobilisation in a halo jacket. No neurological deterioration occurred. The halo brace device therefore does not provide complete mechanical stability in all patients. We now believe, however, that we can select the patients in whom this is likely to happen. Patients with severe vertebral burst fractures or anterior wedging accompanied by marked inter-spinous widening will have disrupted posterior ligamentous complexes. In these patients we electively restore posterior stability by spinous process wiring before mobilisation in the halo jacket. There have been 8 such operations on those treated with the halo brace compared to 5 in the series treated by caliper traction and bed rest. No patient has deteriorated following a stabilisation operation. The skin under the jacket requires attention to prevent pressure sores, especially over the scapulae in thin patients. With careful management we have only had 2 patients with superficial grazes. 
Patients treated with calipers have a prolonged period of bed rest with inherent risks such as boredom, disorientation, pressure sores and weight loss including loss of functional muscle bulk. These patients often start active rehabilitation with low morale at about 2 months and get their first weekend at about 10 to 14 weeks post-injury.

\section{Conclusion}

We therefore believe that the halo brace device for patients with cervical spinal cord injury affords significant physical, psychological and management benefits over traditional skull calipers and bed rest.

\section{References}

Chan RC, Schweigel JF, Thompson GB 1983 Halo thoracic brace immobilisation in 188 patients with acute cervical spina injuries. Fournal of Neurosurgery 58:508-515.

Cooper PR, Maravilla KR, Sklar FH, Moody SF, Kemp Clark W 1979 Halo immobilisation of cervical spine fractures. Fournal of Neurosurgery 50:603-610.

JAMES JIP 1980 Fracture dislocation of the cervical spine. Fournal of the Royal College of Surgeons (Edin) 5:232-233.

PERRY J, NICKEL VL 1959 Total cervical spine fusion for neck paralysis. Fournal of Bone and foint Surgery 41(A):37-60.

Tator CH, Ekong CEU, Rowed DW, Schwartz ML, Edmonds VE 1982 Halo devices for the treatment of cervical spinal cord injuries. In: Early management of acute spinal cord injury. Tator CH (ed.), Raven Press NY. 